\title{
Another piece of the prion puzzle
}

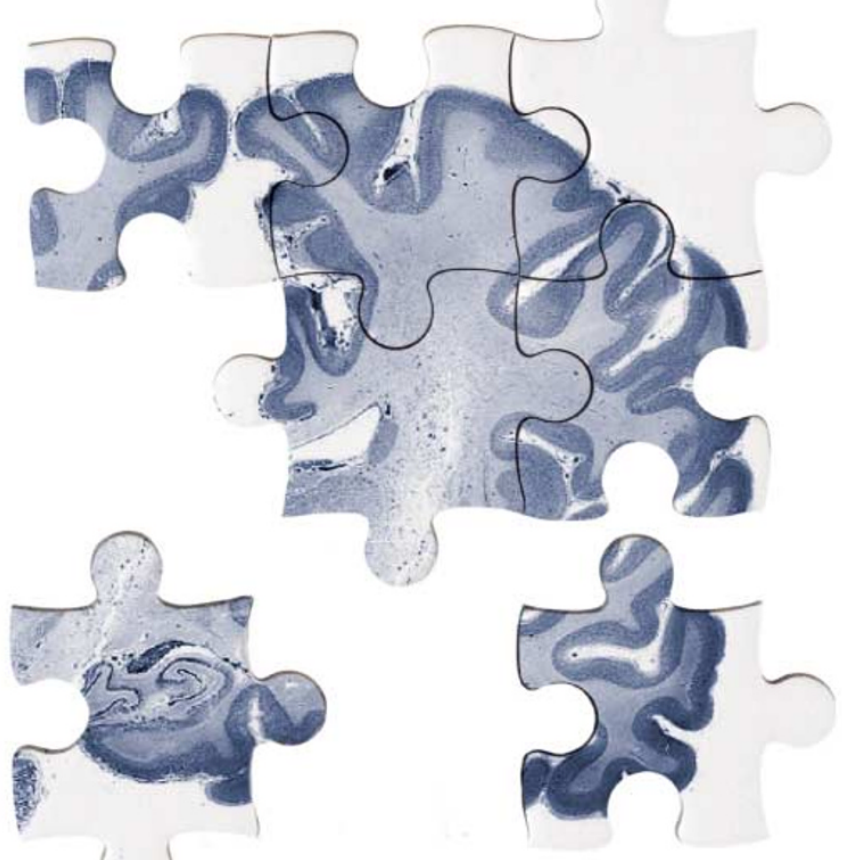

The prion hypothesis - that spongiform encephalopathies are caused and transmitted by a misfolded prion protein - has been accepted for some time, but has not yet been proved. Now, research published in Science takes us a step closer to proving the infectivity of prion protein. Legname et al. report that synthetic mammalian prions cause disease when they are transferred to transgenic mice.

Legname et al. used Escherichia coli to produce recombinant mouse prion protein (recMoPrP), which they polymerized into amyloid fibrils, creating the synthetic prions. Intracerebral injection was used to administer these synthetic prions to transgenic mice that expressed very high levels of cellular prion protein $\left(\operatorname{PrP}^{\mathrm{C}}\right)$. The mice subsequently developed neurological disease, although with a long incubation period of between 380 and 660 days. Control mice, inoculated with phosphate-buffered saline, did not develop symptoms of neurological dysfunction after 620 days.

The authors then took prions from the brains of mice infected with the synthetic prion and transferred them to transgenic and wild-type mice. This resulted in disease in both types of mice, and, although the incubation period was longer in wild-type mice, the incubation period in all the mice was considerably shorter than that of the mice that received the original synthetic prions.

Legname et al. point out that as the amino-acid sequence used in creating the synthetic prion did not contain the asparagine-linked

\section{New players in presynaptic organization}

One of the most intriguing questions in developmental neuroscience is: how are growth cones transformed into presynaptic terminals? Retrograde signals from the postsynaptic cell are believed to be instrumental in this process, and a new study from the laboratory of Joshua Sanes has uncovered an important role for members of the fibroblast growth factor (FGF) family in presynaptic organization.

Umemori et al. prepared an extract from postnatal day 7 mouse forebrains, and screened this extract for molecules that could induce clustering of synaptic vesicles in the neurites of cultured chick motor neurons. They purified one of the main components of the extract that promoted vesicle clustering, and identified this component as FGF22. The FGF22-induced vesicle clusters underwent recycling in response to neuronal depolarization, implying that FGF22 was promoting the development of functional presynaptic structures.

The authors then went on to investigate whether FGF22 promotes presynaptic differentiation in vivo, and they obtained several lines of evidence to indicate that this is indeed the case. FGF22 is expressed in cerebellar granule cells at the same time that they are being innervated by mossy fibres from vestibular and pontine neurons, and, in turn, the vestibular and pontine neurons express the FGFR2 gene, which codes for the main FGF22 receptor FGFR2b. Mossy-fibre presynaptic differentiation was impaired both in vivo and in vitro when FGF22 activity was neutralized with a soluble recombinant form of FGFR2b (FGFR2bAP), and conditional deletion of the FGFR2 gene in newborn mice produced a similar result.

FGF22 belongs to an FGF subfamily that also includes FGF7 and FGF10. All three molecules were shown to promote vesicle clustering in vitro, and all three bind to FGFR2b and are neutralized by FGFR2bAP. Therefore, the authors could not rule out the possibility that FGF7 and FGF10 were responsible for the effects that they observed in vivo. However, FGF22 is expressed at a much higher level in the cerebellum than either FGF7 or FGF10, so it seems likely that it is the main presynaptic organizing molecule, at least in this part of the brain.

Through this work, Umemori et al. have not only defined a new role for postsynaptically derived FGFs in presynaptic assembly, but they have also assigned a developmental function to the FGF7/10/22 subfamily, which has not previously been implicated in neural development. The next step will be to show by more direct means that FGF22 induces presynaptic differentiation in vivo, and mice with mutations in the FGF22 gene are already being generated to test this hypothesis. It will also be interesting to find out whether FGF7 and FGF10 have similar roles at other types of synapse.

Heather Wood

(2) References and links ORIGINAL RESEARCH PAPER Umemori, H. et al. FGF22 and its close relatives are presynaptic organizing molecules in the mammalian brain. Cell 118, 257-270 (2004) FURTHER READING Ziv, N. E. \& Garner, C. G. Cellular and molecular mechanisms of presynaptic assembly. Nature Rev. Neurosci. 5, 385-399 (2004) 
oligosaccharides or the glycosylphosphatidylinositol anchor of the prion, these parts are not required for infectivity, although this does not mean that they have no influence on infectivity.

The authors conclude that all that seems to be required for the spontaneous formation of prions in any mammal is host prion protein. They propose that no additional agent is needed, which would explain the pathogenesis of sporadic CreutzfeldJakob disease. However, tangible evidence to back up these theories and prove the prion hypothesis requires further research. The real test is whether synthetic prions can cause disease when injected into wild-type mice, which express a much lower level of $\mathrm{PrP}^{\mathrm{C}}$ than the transgenic mice used here.

Sarah Archibald

6) References and links ORIGINAL REFERENCE PAPER Legname, G. et al. Synthetic mammalian prions. Science $\mathbf{3 0 5}$, 673-676 (2004)

FURTHER READING Aguzzi, A., Glatzel, M. Montrasio, F., Prinz, M. \& Heppner, F. L. Interventional strategies against prion diseases. Nature Rev. Neurosci. 2, 745-749 (2001)

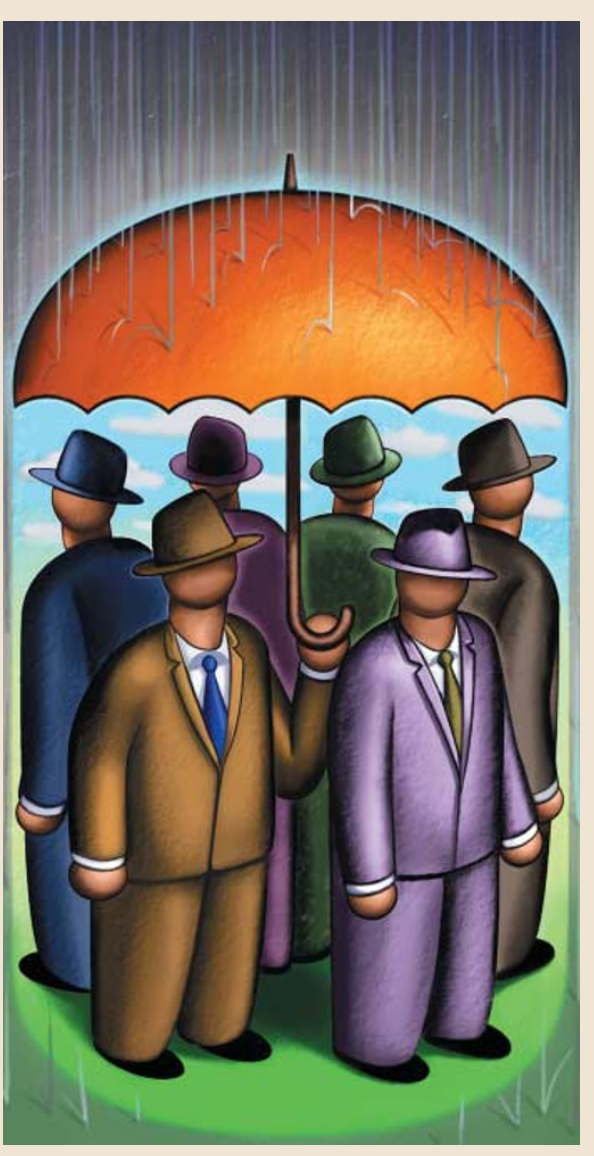

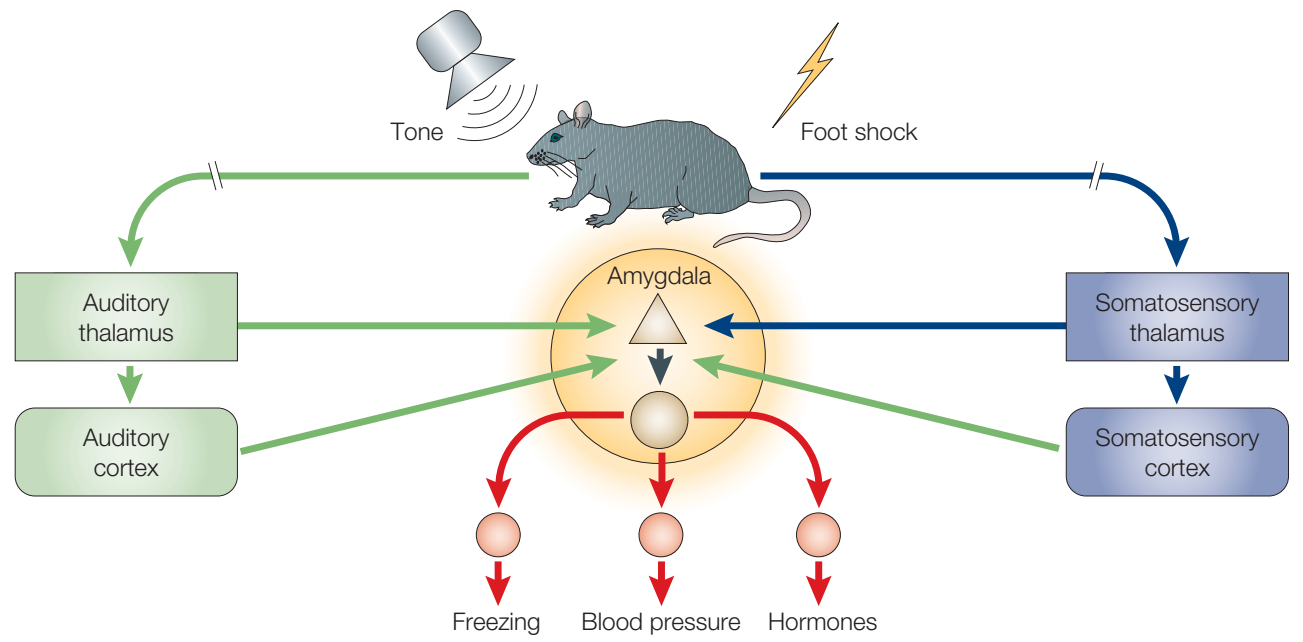

LEARNING AND MEMORY

\section{Learning to fear}

A new study, published in the European Journal of

Neuroscience, shows that NMDA ( $N$-methyl-Daspartate) receptors are essential for the acquisition of both fear conditioning and the associated neurophysiological changes in the lateral amygdala, but not for the expression of these changes.

In Pavlovian fear conditioning, animals learn to associate a conditioned stimulus (for example, a tone) with an aversive, unconditioned stimulus (such as a shock). After a single training trial (one experience of the paired stimuli), the conditioned stimulus itself becomes able to elicit fear responses, such as increases in heart rate or freezing. Because learning in this protocol takes just one session, it is ideal for differentiating the mechanisms of learning from those of expression (or remembering).

Long-term potentiation - a cellular model of associative learning - occurs in the amygdala and requires NMDA-receptor activation, and NMDAreceptor blockade can attenuate fear learning in conditioned fear experiments. However, pharmacological manipulation of other receptors in the amygdala, such as metabotropic glutamate receptors or AMPA ( $\alpha$-amino-3-hydroxy-5methyl-4-isoxazole propionic acid) receptors, also alters fear conditioning, making it unclear whether plasticity in the amygdala related to fear conditioning is mediated solely by NMDA receptors. So Goosens and Maren investigated the effects that blocking NMDA receptors had on neuronal plasticity in the amygdala of awake, behaving rats, and the effects on both learning and expression of conditioned fear. They used a competitive NMDA-receptor antagonist, CPP ( \pm 3 -(2-carboxypiperazin-4-yl) propyl-1phosphonic acid), and single-unit recording of neurons in the lateral amygdala.

When rats were treated with CPP just before the training session, they did not subsequently show conditioned fear responses, such as freezing, to the conditioned stimulus. Unlike control rats, treated rats also showed no significant increase in neuronal spike firing in the lateral amygdala in response to the conditioned stimulus after training. These results support the idea that NMDA receptors are required for acquisition of both fear conditioning and associative plasticity in the lateral amygdala.

To investigate the role of NMDA receptors in the expression of conditioned fear and associative plasticity, Goosens and Maren treated rats with CPP just before testing, rather than before training. This treatment reduced the amount of response to the conditioned stimulus, but did not completely abolish it. By contrast, CPP treatment did not affect the expression of associative plasticity in the lateral amygdala — neurons here still showed increased firing in response to the conditioned stimulus, regardless of whether the rats had been treated with CPP.

So, non-NMDA receptors in the amygdala seem not to be able to support either conditioned fear learning or associative neuronal plasticity after training, but they are sufficient for the expression of the conditioned fear response (albeit reduced) and of associated spike firing. Because CPP has a particular affinity for the NR2A and NR2B subunits, it would seem that NMDA receptors containing these subunits are important for conditioned fear learning and for the plasticity that is associated with it.

Rachel Jones

(2) References and links

ORIGINAL RESEARCH PAPER Goosens, K. A. \& Maren, S. NMDA receptors are essential for the acqisition, but not expression, of conditional fear and associative spike firing in the lateral amygdala. Eur. J. Neurosci. 20, 537-548 (2004)

FURTHER READING Medina, J. F. et al. Parallels between Neurosci. 3, 122-131 (2002)

WEB SITE

Maren's laboratory: http://marenlab.org/ conditioned fear behaviour shown by the rats in

cerebellum- and amygdala-dependent conditioning. Nature Rev. 